\title{
FATORES QUE AFETAM A FIXAÇÃO DO FÓSFORO II. CONCENTRAÇÃO DA SOLUÇÃO ADICIONADA *
}

\author{
Francisco José de Albuquerque Cavalcanti ** \\ Francisco de Assis Ferraz de Mello***
}

\section{RESUMO}

Foi feito um estudo sobre fixação de $\mathbf{P}$ utilizando soluções de fosfato monocálcio e solos que ocorrem na região de Piracicaba, São Paulo. Dos solos utilizados, 3 são Latossolos (Solos I, II e V) e dois Pdzólicos (Solos III e IV).

O período de incubação estabelecido para cada tratamento $\left(P_{1}=500 ; P_{2}=1.000 ; P_{3}=1.500 \mathrm{ppm}\right.$ de $\left.P\right)$ foi de 200 horas.

Cada unidade experimental constou de um recipiente contendo $10 \mathrm{~g}$ de terra que recebeu $4 \mathrm{ml}$ de solução.

A determinação do teor de $\mathrm{P}$, no final do ensaio, foi feita no extrato obtido com uma solução $0,025 \mathrm{~N}$ em $\mathrm{H}_{2} \mathrm{SO}_{4}$ e $0,05 \mathrm{~N}$ em $\mathrm{HCl}$.

A fim de se proceder à correção dos dados inicialmente encontrados foi preciso tratar cada amostra de solo com $4 \mathrm{ml}$ de água destilada $\left(\mathrm{P}_{0}\right)$, submetendo-a ou não ao período de incubação estabelecido.

A quantidade de $P$ fixada foi obtida subtraindo-se do "extrato corrigido" do material não submetido à incubação os valores corrigidos de $\mathrm{P}_{1}, \mathrm{P}_{2}$ e $\mathrm{P}_{3}$, após o período de incubação.

Constatou-se, para todos os solos, efeitos linear e quadrático de tratamentos altamente significativos. Os Latossolos apresentaram maior poder de fixação de fosfato que o Podzólico número IV.

\section{INTRODUÇÃO}

A quantidade de fósforo fixada por um solo está na dependência de diversos fatores. Assim, por exemplo, a retenção poderá ser mais ou menos acentuada de acordo com a textura, o tipo de argila, o pH, etc.

* Trabalho realizado com parte dos dados contidos na Dissertação apresentada pelo primeiro autor à E.S.A. "Luiz de Queiroz", USP, em 1974. Entregue para publicação em 27-12-77.

** Eng. ${ }^{\circ}$ Agr. ${ }^{\circ}$, Mestre em Solos e Nutrição de Plantas, da Seção de Solos do Instituto de Pesquisa Agropecuária do Nordeste (IPEANE), Recife, Pernambuco.

*** Prof. Adjunto do Departamento de Solos, Geologia e Fertilizantes, ESALQ, USP. 
Os argilo-minerais concorrem para a fixação do fósforo até pela liberação do alumínio (COLE \& JACKSON, 1950), de vez que esse elemento na forma de alumínio $\mathrm{Al}^{+3}$ trocável é bastante ativo na precipitação de fosfatos.

Por outro lado, a presença de óxidos hidratados cristalinos e/ou amorfos de $\mathrm{Fe}$ e $\mathrm{Al}$ responde, em parte, pela retenção do fósforo em solos intensamente intemperizados.

A presença de matéria orgânica, desde que o contacto com o solo não seja muito demorado (AHMAD MOSTAFA, 1969) poderá reduzir a intensidade do processo de fixação. Tanto assim que HUDCOVA \& KOVAROVA (1963) assinalaram um aumento na absorção do fósforo por partículas coloidais ao se proceder o seu tratamento com água oxigenada.

Por sua vez, o efeito da concentração do íon fosfato de uma solução adicionada ao solo na quantidade de fósforo fixada - objetivo deste trabalho - vem sendo também pesquisado pelos que se dedicam ao assunto.

A importância desse último fator é ressaltada por LARSEN (1967) quando relaciona a adsorção do fósforo com a sua precipitação. Para esse autor, a precipitação desse elemento só ocorre quando o sistema responsável pela adsorção atinge o ponto de saturação. Em outras palavras, a solubilidade dos compostos precipitados dependerá de concentração do fósforo na solução.

No final de um período de incubação de 200 horas foi efetuado um estudo acêrca da quantidade de fósforo fixada por amostras da camada arável de cinco Séries de solo que ocorrem na região de Piracicaba, São Paulo, fazendo-se variar apenas a concentração da solução de fosfato monocálcico utilizada.

\section{MATERIAL E METODOS}

A amostra da camada arável correspondente ao Solo I (Quadro I) foi coletada nas imediações do perfil estudado por ANDRADE (1971) sob a denominação de $\mathrm{P}_{2}$, enquanto que as de número II, III, IV e V foram tomadas, respectivamente, ao lado dos perfis $P_{20}, P_{26}, P_{28}$ e $P_{33}$ constantes da Carta de Solos do Município de Piracicaba (RANZANI et $a l ., 1966)$. Os trabalhos de laboratório foram desenvolvidos no Departamento de Solos e Geologia da Escola Superior de Agricultura "Luiz de Queiroz" da Universidade de São Paulo (ESALQ-USP) .

Antes de analisadas (Quadros 2 e 3), as amostras foram secadas ao ar e passadas em peneiras de $2 \mathrm{~mm}$. Para cada tratamento tomou-se o equivalente a $10 \mathrm{~g}$ de TFSE, onde foram adicionados $4 \mathrm{ml}$ de uma solução de $\mathrm{Ca}\left(\mathrm{H}_{2} \mathrm{PO}_{4}\right)_{2}$, de concentração conhecida (WAUGH \& FITTS, 1966) . 
QUADRO 1 - Classificação dos solos utilizados (Adaptado de RANZANI et al. 1966)

\begin{tabular}{rlll}
\hline & & \multicolumn{2}{c}{ Grande Grupo } \\
\cline { 3 - 4 } Amostra & Série & $\begin{array}{c}\text { (Comissão de } \\
\text { Solos CNEPA) }\end{array}$ & $\left(7 .^{\text {a }}\right.$ Aproximação $)$ \\
\hline I & Guamium & Latossolo & Haplacrox \\
II & Iracema & Latossolo & Haplacrox \\
III & Gibóia & Podzólico & Typustalf \\
IV & Ibitiruna & Podzólico & Typochrult \\
V & Luiz de Queiroz & Latossolo & Ultustalf \\
\hline
\end{tabular}

QUADRO 2 - Análise mecânica dos solos utilizados

\begin{tabular}{ccccc}
\hline & \multicolumn{3}{c}{ Porcentagem } & \\
\cline { 2 - 4 } Amostra & Areia & Limo & Argila & Classe \\
& $\begin{array}{c}(2-0,05) \\
\mathrm{mm}\end{array}$ & $\begin{array}{c}(0,05-0,002) \\
\mathrm{mm}\end{array}$ & $\begin{array}{c}(<0,002) \\
\mathrm{mm}\end{array}$ & Textural \\
\hline I & 19,8 & 17,3 & 62,9 & \\
II & 23,7 & 30,4 & 45,9 & Argiloso \\
III & 73,3 & 20,2 & & Argiloso \\
IV & 88,0 & 5,8 & 6,5 & Arenoso \\
V & 35,3 & 26,0 & 6,2 & franco \\
& & & 38,7 & Arenoso \\
\hline
\end{tabular}

Foram estabelecidas 3 concentrações $\left(P_{1}=500, P_{2}=1.000 \mathrm{e}\right.$ $\mathrm{P}_{3}=1.500 \mathrm{ppm}$ de P) para a solução de fosfato monocálcico. Apesar do período de incubação escolhido ter sido 200 horas, determinou-se também o teor de fósforo do material não submetido à incubação.

O controle das perdas de água foi feito pelas alterações de peso constatadas durante todo o ensaio. Ao término do periodo de incubação determinaram-se os teores de fósforo dos extratos obtidos com uma solução $0,025 \mathrm{~N}^{2} \mathrm{em} \mathrm{H}_{2} \mathrm{SO}_{4}$ e $0,05 \mathrm{~N}$ em $\mathrm{HCl}$ (VETTORI, 1966).

A quantidade de fósforo fixada foi obtida, em cada tratametno, subtraindo-se do "extrato corrigido" do material não submetido à incubação as respectivas concentrações corrigidas de $P_{1}, P_{2}$ e $P_{3}$ após 0 tempo de contacto de 200 horas .

A fim de se proceder a correção dos dados inicialmente encontrados foi preciso tratar cada amostra de solo, durante 0 e 200 horas, com $4 \mathrm{ml}$ de água destilada $\left(\mathrm{P}_{0}\right)$.

As quantidades de fósforo fixadas foram submetidas a uma análise estatística, levando-se em conta cada solo de per si, e a uma análise conjunta que permitiu fazer comparações entre os solos estudados. 


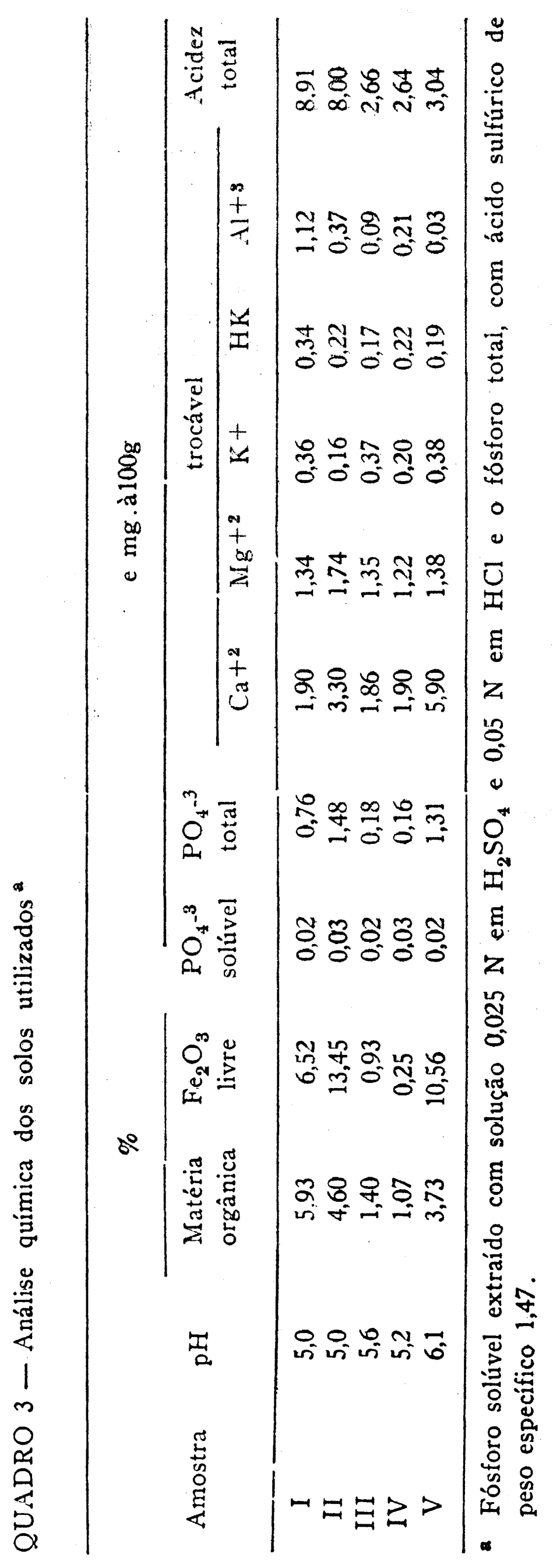




\section{RESULTADOS E DISCUSSÃO}

A partir da concentração dos extratos obtidos, tanto para o material que não foi submetido à incubação como para aquele em que o tempo de contacto atingiu 200 horas (Quadros 4 e 6, respectivamente), foi possível a confecção dos Quadros 5 e 7, "extratos corrigidos".

No Quadro 8 podem ser vistas as quantidades de fósforo fixado, para cada solo e concentração da solução de fosfato monocálcico.

Para facilitar a comparação entre os comportamentos dos solos utilizados foi feito um gráfico, relacionando-se a quantidade média de fósforo fixado para cada solo com a concentração da solução adicionada (Fig. 1).

A análise estatística dos resultados obtidos para cada solo revelou a ausência de diferença significativa ao nível de 5\%, pelo teste de Tukey, entre os períodos de incubação $P_{1}$ e $P_{2}$ para o Solo IV. Quanto ao restante das amostras, houve sempre diferenças significativas, ao nível de $5 \%$, para os tratamentos empregados.

Desdobrando-se os graus de liberdade obteve-se, para todos os solos, uma significância estatística ao nível de $1 \%$ para os efeitos linear e quadrático da concentração da solução adicionada (Quadro 9) .

Pela análise conjunta para os solos I, II, IV e V (Quadro 10) constatou-se, através do teste de Tukey que, ao nível de $5 \%$, os Latossolos (Solos I, II e V) diferiam significativamente do solo Podzólico de número IV. Devido ao valor elevado do quadrado médio do resíduo, o Solo III não pôde sẹ incluido nessa análise.

Por outro lado, as quantidades de fósforo fixadas pelos solos em estudo, acham-se bastante relacionadas com algumas de suas características. Desse modo, o Solo IV (Podzólico) que fixou uma quantidade de fósforo inferior à retida pelos Solos I, II e V (Latossolos) apresenta-se, com um teor de areia igual a 88,0\% enquanto que os teores encontrados para o Latossolos variaram de 19,8 a $35,3 \%$.

Por sua vez, as porcentagens de $\mathrm{Fe}_{2} \mathrm{O}_{3}$ livre estão de acordo com os resultados da análise estatística para os quatro solos em conjunto, ou seja, valores elevados para os Latossolos (Solo I $=6,52$; Solo II $=13,45$ e Solo $\mathrm{V}=10,56 \%$ ), ao passo que só atingiu $0,25 \%$ para o Solo IV (Podzólico) . 


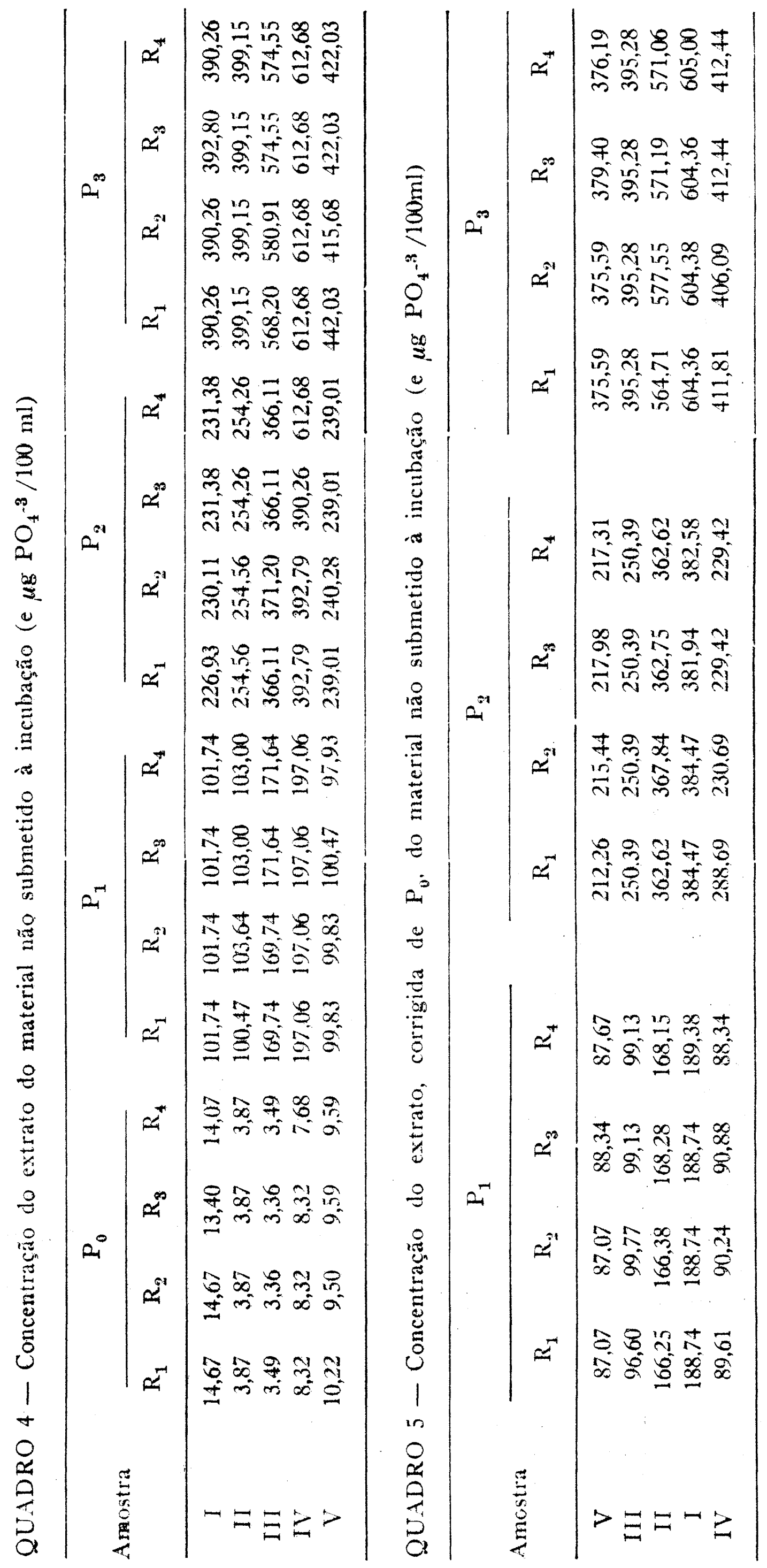




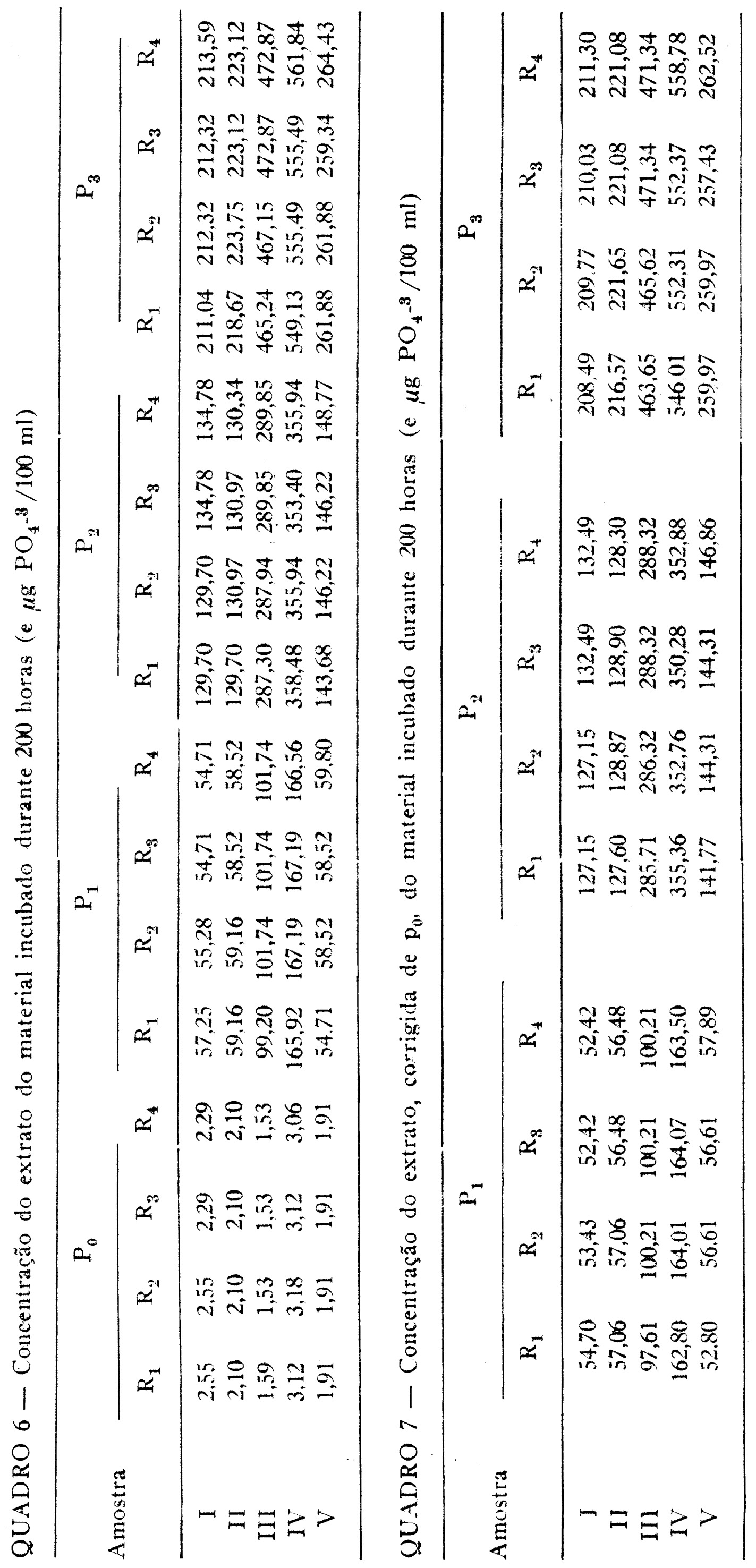




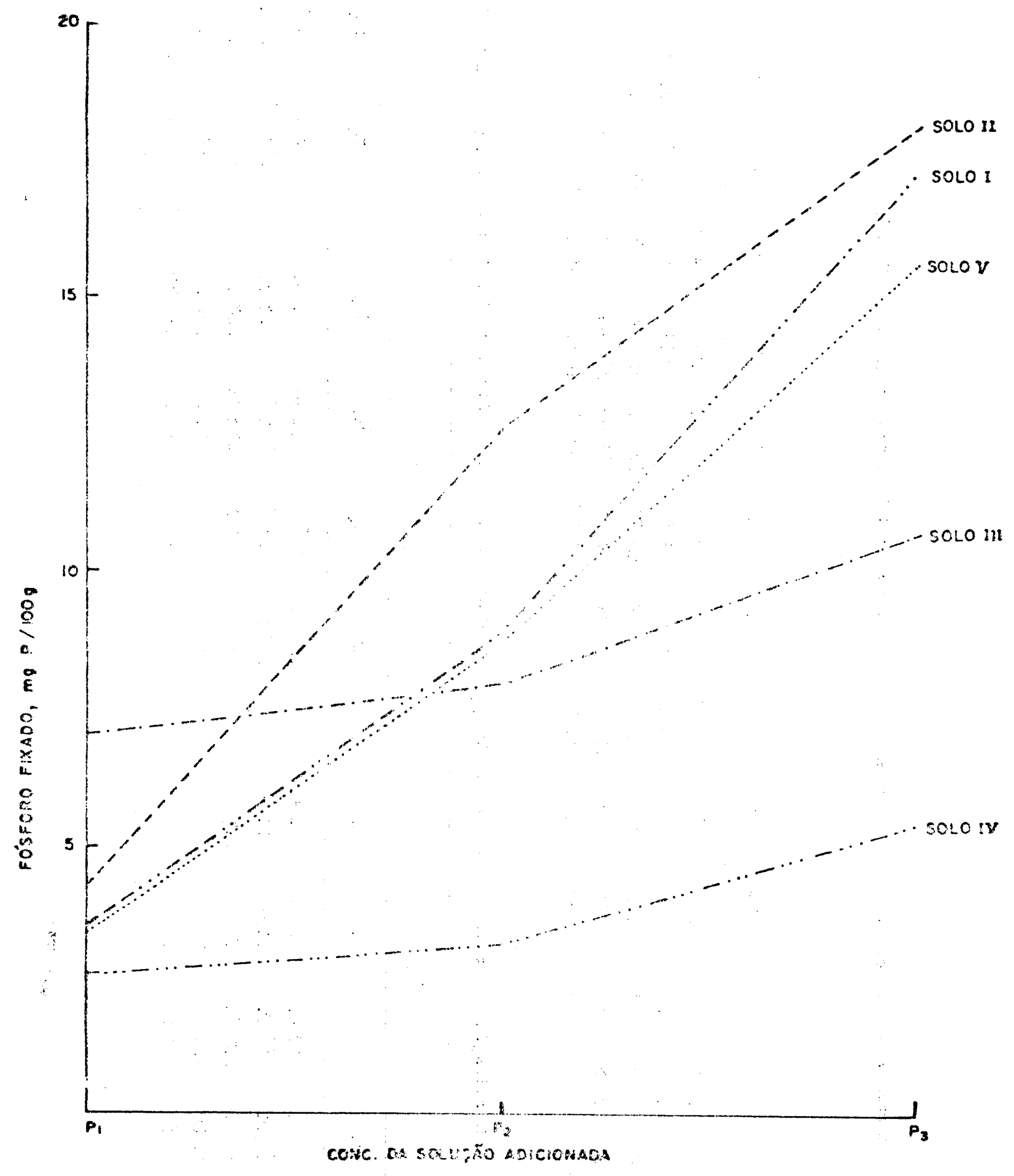

Figura 1 - Quantidade média de fósforo fixado 


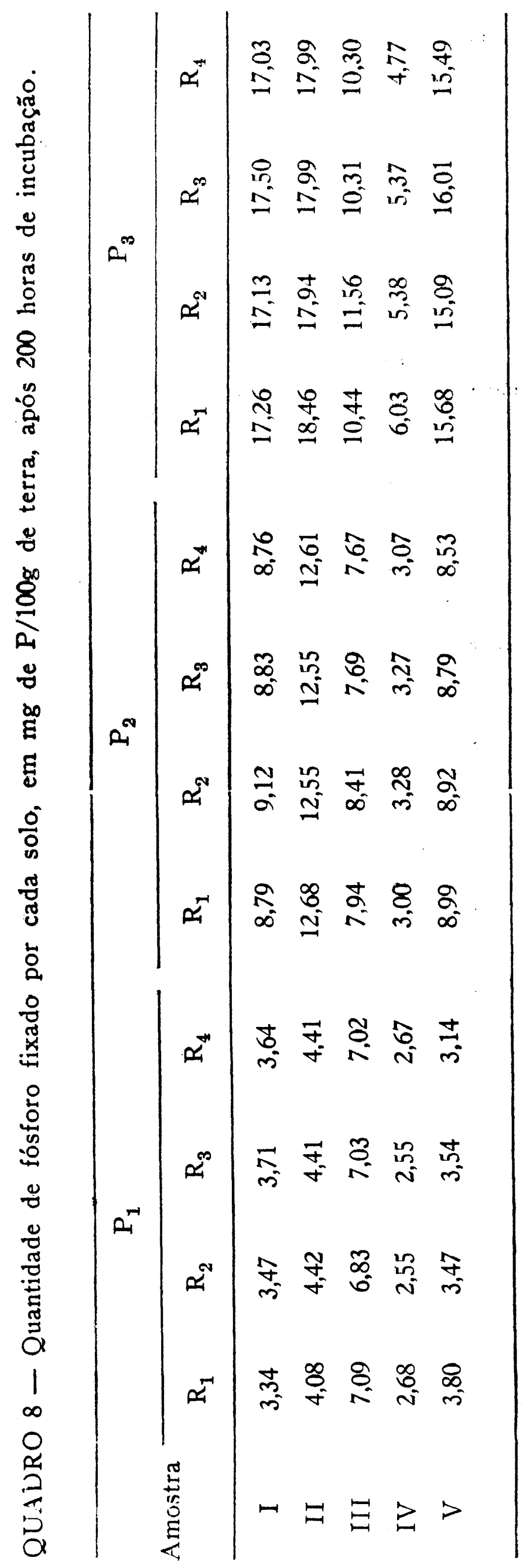


QUADRO 9 - Análise da variância

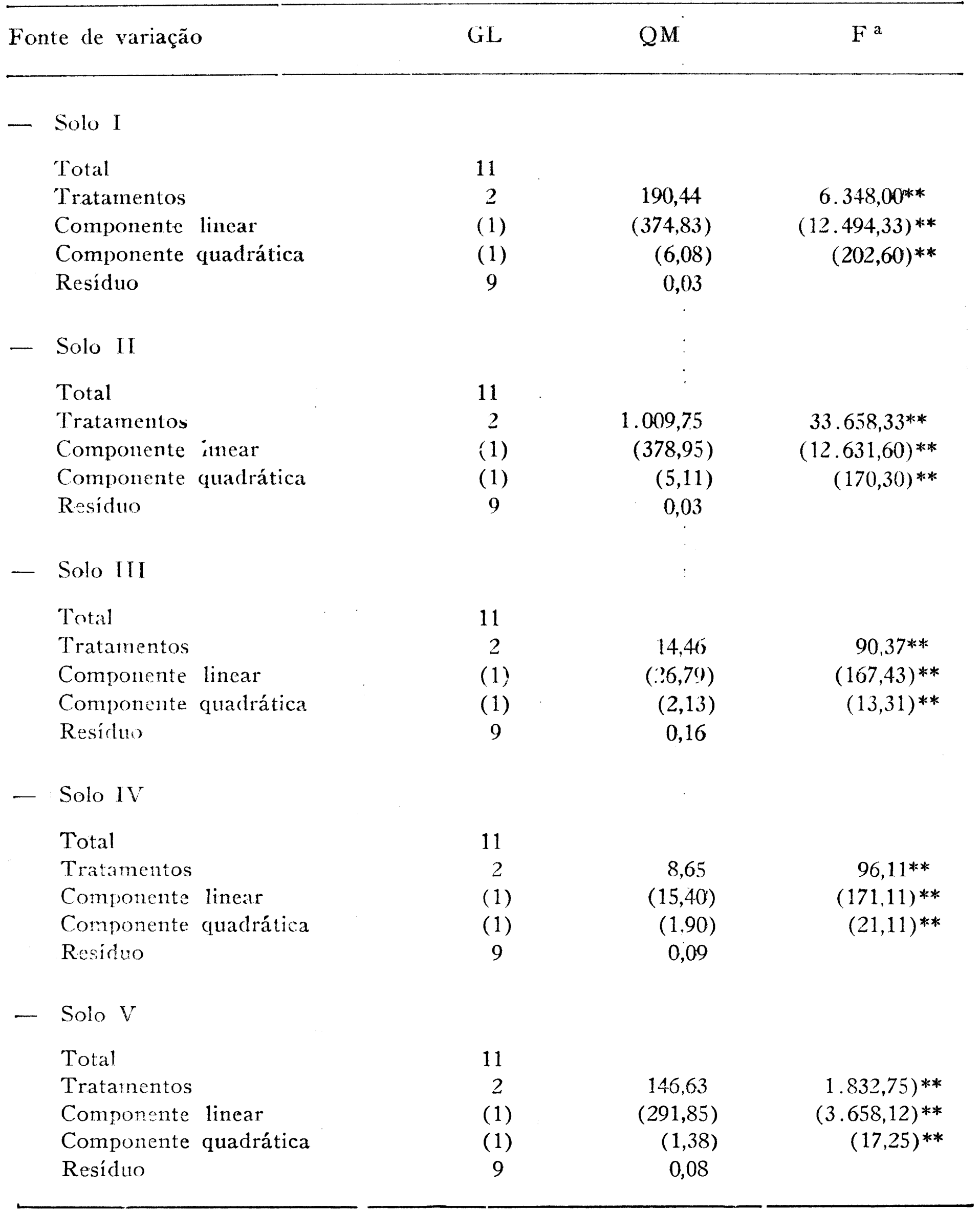

$a^{* *}=$ Singficiativo ao nível ie $1 \%$. 
QUADRO 10 - Análise da variância (Solos I, II, IV e V)

Fonte de variação

GL

QM

$\mathrm{F}^{\mathrm{a}}$

Total

Blocos

12

Tratamentos

3

2

Resicho

7

35,43

$5,56^{*}$

112,07

$17,59 * *$

$a^{*}=$ significativo ao nível de $5 \%$

** = significativo ao nível de $1 \%$

OBS.: d.m.s. ao nível de $5 \%$ pelo teste de Tukey $=5.25$

Solo $\mathrm{I}=9,88$

Solo II $=11,67$

Solo IV $=3,71$

Solo $\mathrm{V}=9,28$

\section{CONCLUSÕES}

Para as condições presentes neste trabalho, e de acordo com a análise estatística dos resultados obtidos, pôde-se tirar as conclusões abaixo:

a) excetuando-se o Solo IV, onde, pelo teste de Tukey ao nível de $5 \%, \mathrm{P}_{1}$ e $\mathrm{P}_{2}$ não se diferenciaram entre sí, todos os outros apresentaram diferenças significativas entre os tratamentos;

b) as concentrações estabelecidas proporcionaram, para todos os solos, a obtenção de significância estatística para os efaitos linear e quadrático, ao nível de $1 \%$;

c) os Solos I, II e V (Latossolos), sem chegar a diferenciarem-se entre si, fixaram uma quantidade de fósforo superior à retida pelo Solo IV (Solo Podzólico), pelo teste de Tukey ao nível de 5\%.

\section{SUMMARY}

\section{FACTORS AFFECTING PHOSPHORUS FIXATION \\ II - CONCENTRATION OF ADDED SOLUTION}

Using four concentrations of monocalcic phosphate solution in soil samples from five different soil series taken from the region of Piracicaba, State of São Paulo, Brasil, a study on phosphorus fixation was carried out. 
Of the soils used, three are Latosols (Soils I, II and V) whereas the rest (Soils III and IV), Podzolics.

1s was observed a 200 hours incubation period for all treatments $\left(\mathrm{P}_{1}=500 ; \mathrm{P}_{2}=\right.$ 1,000 and $P_{3}=1,500 \mathrm{P}$ ppm).

Each experimental unity consisted of a container with $10 \mathrm{~g}$ of soil which received $4 \mathrm{ml}$ of the mentioned solution. At the end of the trial, the evaluation of phosphorus content was done with an extractable solution of $0.025 \mathrm{~N} \mathrm{H}_{2} \mathrm{SO}_{4}$ and $0.05 \mathrm{~N} \mathrm{HCl}$.

In order to make corrections on original data each soil sample received a $4 \mathrm{ml}$. destilled water treatment $\left(\mathrm{P}_{0}\right)$ submitted or not to the 200 hour incubation period. The quantity of fixed phosphorus was calculated by subtracting the values found for $\mathrm{P}_{1}, \mathrm{P}_{2}$ and $P_{3}$ those submitted to the 200 hour incubation period from the values for the "corrected strata", those not submitted to the incubation period.

Statistical analysis of data allow the following statments:

Linear and quadratic effects proved to be highly significant in all soils studied;

Latosols fixed more phosphorus than Soil IV. Soil III could not be included in the joint analysis because of its high means square error.

\section{LITERATURA CITADA}

AHMAD MOSTAF, E.S., 1969. Movement of phosphorus in soils as influenced by chelates and soil types. Diss. Abst. 30(3):937-938. In Phosphorus in Agricult. Abst. n. ${ }^{\circ}$ 55:81. 1970.

ANDRADE, S.S. de, 1971. Gênese e classificação de solos de três catenas nós municípios de Piracicaba e Rio Claro. Dissertação Mestrado, Piracicaba, E.S.A. "Luiz de Queiroz", 74 p. (Mimeografada).

CAVALCANTI, F.J. de A., 1974. Alguns aspectos da fixação do fósforo por solos na região de Piracicaba. Dissertação Mestrado, Piracicaba, E.S.A. "Luiz de Queiroz" - USP. 62 p. (Mimeografada)

COLF, C.V. \& M.L. JACKSON, 1950. Solubility equilibrium constant of dihydroxy phosphate relating to a mechanism of phosphate fixation in soils. Soil Sci. Soc. Amer. Proc. 15: 84-88.

HUDCOVA, O. \& B. KOVAROVA, 1963. The sorption of phosphorus by clay minerals. Rostl. Vorab 15:1067-1073. In Phosphorus in Agriculture Abst. n. ${ }^{\circ}$ 56: 49.1970 .

LARSEN, S, 1967. Soil phosphorus. Advances in Agronomy 19: 151-207.

RANZANI, G., O. FREIRE \& T. KINJO, 1966. Carta de Solos do Municipio de Piracicala. Centro de Estudos de Solos da E.S.A. "Luiz de Queiroz", USP, 85 p.

VETTORI, L., 1966. Métodos de análise de solos. Divisão de Pedologia e Fertilidade do Solo. Rio de Janeiro, 19 p. (Mimeografado).

WAUGH, D.L. \& J.W. FITTS, 1966. Estudos para interpretação de análises de solo; de laboratório e de vasos. Boletim Técnico n. ${ }^{\circ} 3$. International soil testing. $33 \mathrm{p}$. 